\title{
An Integrated SiGe Dual-band Low Noise Amplifier for Bluetooth, HiperLAN and Wireless LAN Applications
}

\author{
Mou Shouxian, Ma Jianguo, Yeo Kiat Seng, and Do Manh Anh
}

Centre for Integrated Circuits \& Systems, Nanyang Technological University, Singapore 639798

\begin{abstract}
A bandpass low noise amplifier (LNA), which can simultaneously operate at two different frequency bands $-2.35 \mathrm{GHz}-2.6 \mathrm{GHz}$ and $5 \mathrm{GHz}-6 \mathrm{GHz}$, is presented in this paper. The proposed dual-band LNA can be used for the Bluetooth, HiperLAN and Wireless LAN (IEEE 802.11a) communication applications, and is designed based on IBM $0.25 \mu \mathrm{m}$ SiGe BiCMOS technology, which has better performance comparing with silicon technology, while lower cost and better integration feasibility comparing with GaAs technology.
\end{abstract}

\section{INTRODUCTION}

Wireless services industry has experienced a tremendous growth in the past few years [1-2]. Mobile cellular and home cordless telephones are fast becoming a part of our daily lives. The wireless local area network (WLAN) communication and home RF network are becoming popular as well. Fig. 1 illustrates the receiving band distribution of current wireless communication standards in the range of $2.4 \mathrm{GHz}-6 \mathrm{GHz}$. With so many communication standards, one may consider to develop multi-functional devices, which can operate at several bands and different modes. However, traditional multifunction device is often bulky and power hungry in general, which limits the size, weight and the battery life of the wireless communication devices [3-5]. Thus, development of a single-chip multi-function $\mathrm{RF}$ receiver with low-cost, low-power and small form-factor characteristics is in demand [4-5].

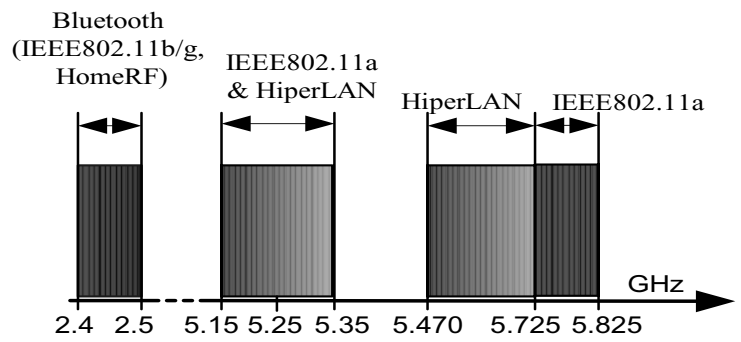

Fig. 1. Receiving band distribution of current wireless communication standards in the range of $2.4 \mathrm{GHz}-6 \mathrm{GHz}$

One of the key bottlenecks for the multiple-standard communication devices is to design a single LNA that can operate at different frequency bands, since the LNA is a very important building block of a single-chip RF receiver and locates at the first stage of the receiving path.

Most of the latest LNAs reported in the literature are single-band LNAs. Currently, there are a few dual-band LNAs used for dual-band applications [6-7]. However,

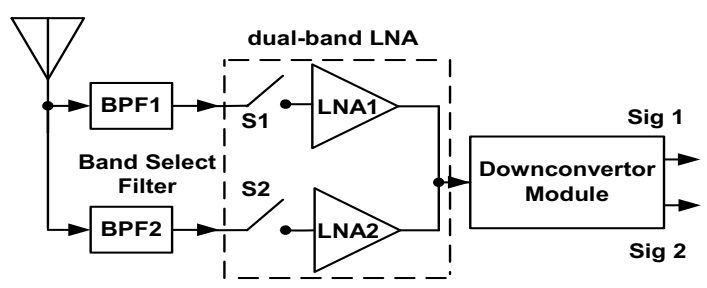

(a)

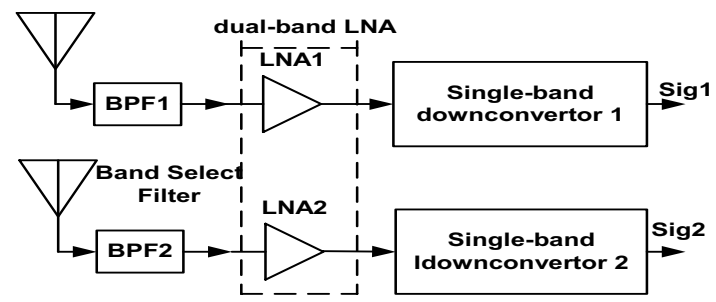

(b)

Fig. 2. Two conventional dual-band LNAs in RF receivers [6-7]

they will cause inevitable increase in the cost, footprint and power dissipation, because each LNA (illustrated in Fig. 2 (a) and (b)) uses two single-band LNAs, either one of which is selected according to the instantaneous band of operation [6], or both of which are designed to work in parallel using two separated input matching circuits and two separated resonant loads [7]. The former approach is non-concurrent while the latter consumes twice as much power.

In this paper, a dual-band bandpass LNA, which can provide simultaneous gains and matching at several frequency bands of interest, will be investigated as one of the alternatives to alleviate the above-mentioned problems.

The proposed LNA is designed to work at $2.35 \mathrm{GHz}$ $2.6 \mathrm{GHz}$ and $5 \mathrm{GHz}-6 \mathrm{GHz}$ for the Bluetooth, HiperLAN and wireless LAN communication applications. The design is based on IBM SiGe $0.25 \mu \mathrm{m}$ BiCMOS technology which offers good performance and high integration feasibility.

\section{INPUT MATCHING}

There are several general considerations in LNA designs, including a $50 \Omega$ input impedance, the minimum noise figure, maximum gain with sufficient linearity and a low power consumption which is very important for portable systems [8-9]. Recent research shows that the optimum input stage topology is the source inductive 
degeneration input stage [9] as described in Fig. 3 (a). Its input impedance can be easily derived,

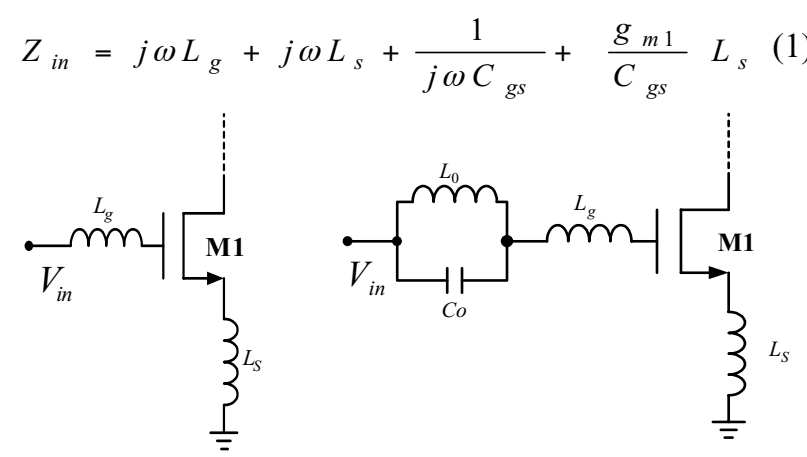

(a)

(b)

Fig. 3. (a) Source inductive degeneration input stage; (b) Input stage for the proposed dual-band LNA in this paper

For input impedance matching purpose, the following equations should be satisfied at the frequency of interest,

$$
\begin{gathered}
\left(\frac{g_{m 1}}{C_{g s}}\right) L_{s}=50 \\
j \omega L_{g}+j \omega L_{s}+\frac{1}{j \omega C_{g s}}=0
\end{gathered}
$$

Solving (3), the resonant/working frequency can be obtained,

$$
\omega=1 / \sqrt{\left(L_{g}+L_{s}\right) C_{g s}}
$$

This is the design key of a single-band LNA's input impedance matching. For dual-band/multi-band LNA, the similar methodology can be adopted. There is only one small change - an additional parallel $L_{0} C_{0}$ network is connected to the gate inductor $L_{g}$, demonstrated in Fig. 3 (b). In terms of its small signal equivalent circuit, the dual-band LNA's input impedance can be expressed as

$$
Z_{\text {in }}=j \omega\left(L_{g}+L_{s}\right)+\frac{1}{j \omega C_{g s}}+\frac{g_{m 1}}{C_{g s}} L_{s}+\frac{j \omega L_{0}}{1-\omega^{2} L_{0} C_{0}}
$$

To obtain a $50 \Omega$ input impedance, the real part and the imaginary part at both resonant frequencies should satisfy:

- Real part:

$$
\left(\frac{g_{m 1}}{C_{g s}}\right) L_{s}=50
$$

- Imaginary part:

$$
j \omega\left(L_{g}+L_{s}\right)+\frac{1}{j \omega C_{g s}}+\frac{j \omega L_{0}}{1-\omega^{2} L_{0} C_{0}}=0
$$

It is obvious that (7) has two solutions at $\omega_{01}$ and $\omega_{02}$. Tuning the circuit to resonate at two interested frequency bands such as $2.4 \mathrm{GHz}$ and $5.8 \mathrm{GHz}$, giving an $L_{s}$ value to satisfy (6), a good input matching for a concurrent dualband LNA for the Bluetooth and WLAN utilizations can be easily realized. The quality of the input matching may greatly influence the overall performance of the designed LNA.

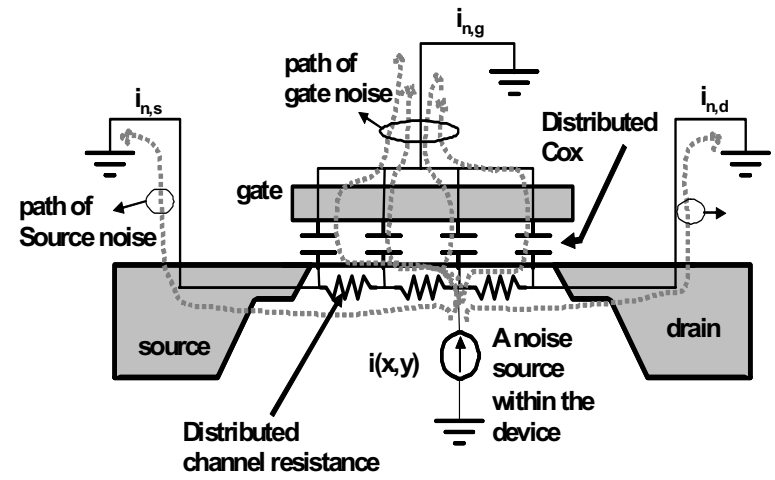

Fig. 4. Description of the noise sources in a MOS transistor [10]

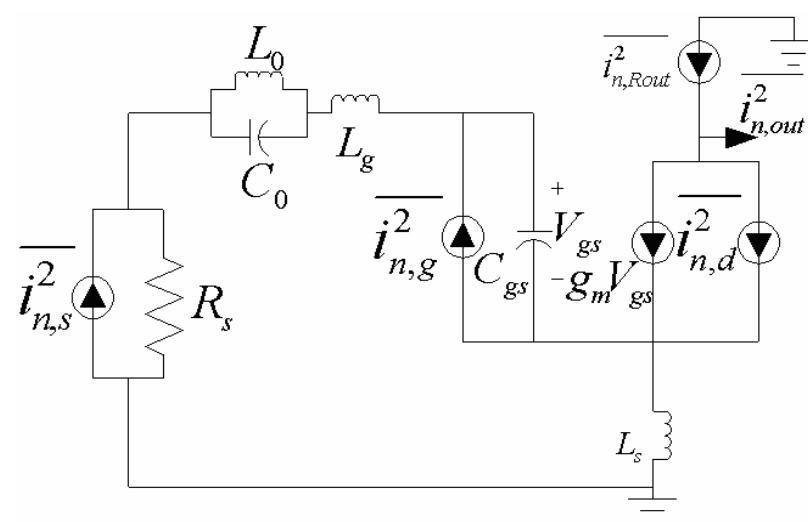

Fig. 5. Equivalent circuit with noise sources in the dual-band LNA

\section{NOISE OPTIMIZATION}

\section{A. Noise Sources}

In a MOS device, the prominent noise sources are drain channel noise $\bar{i}_{n, d}^{2}$, gate induced noise $\bar{i}_{n, g}^{2}$, distributed gate resistance thermal noise, substrate thermal noise and $\overline{i^{2}}$ n,corr , which is due to the correlation between $\bar{i}_{n, g}^{2}$ and $\bar{i}_{n, d}^{2}$. Fig. 4 gives a visual illustration and Fig. 5 depicts an equivalent circuit with noise sources of the proposed dual-band LNA. The noise sources are expressed as follows:

$$
\begin{aligned}
& {\overline{i^{2}}}_{n, R_{s}}=4 k T \frac{1}{R_{s}} \Delta f \\
& {\overline{i^{2}}}_{n, R_{\text {out }}}=4 k T \frac{1}{R_{\text {out }}} \Delta f \\
& \overline{{\overline{i_{n, g}^{2}}}^{2}}=4 k T \delta g_{g} \Delta f \\
& \overline{i_{n, d}^{2}}=4 k T \gamma g_{d 0} \Delta f
\end{aligned}
$$

The definitions of the parameters $\gamma, \delta, g_{d 0}$ and $g_{g}$ can be found in [8, 9 and 11].

\section{B. Output Noise and Noise Optimization}

Through circuit analysis, the transfer functions of the above noise sources to the output noise current (see Fig.5) 
at resonance can be obtained:

$$
\begin{gathered}
i_{n, o, R_{s}}=\frac{g_{m}}{j 2 \omega_{o} C_{g s}} i_{n, R_{s}} \\
\frac{i_{n, o, d}=\frac{1}{2} i_{n, d}}{i_{n, o, R_{\text {out }}}^{2}}=\frac{i_{n, R_{\text {out }}}^{2}}{i_{n, o, g}=\frac{g_{m}}{j \omega_{o} C_{g s}} \frac{j \omega_{o} C_{g s} R_{s}-1}{j 2 R_{s} \omega_{o} C_{g s}} i_{n, g}} \\
\overline{i^{2}{ }_{n, o, c o r r}}=\frac{g_{m}|c|}{2 \omega_{o} C_{g s}} \cdot \sqrt{\overline{i^{2}}}{ }_{n, g} \cdot \overline{i^{2}}
\end{gathered}
$$

Then the noise factor $F$ can be obtained,

$$
\begin{aligned}
& F=\frac{{\overline{i^{2}}}_{n, o, R_{s}}+{\overline{i^{2}}}_{n, o, g}+{\overline{i^{2}}}_{n, o, d}+{\overline{i^{2}}}_{n, o, c o r r}+\bar{i}_{n, o, R_{o u t}}}{{\overline{i^{2}}}_{n, o, R_{s}}} \\
& \approx 1+\frac{\delta \alpha}{5 g_{m} R_{s}}+\left(\frac{\delta \alpha g_{m}}{5 R_{s}}+\frac{\gamma g_{m}}{\alpha R_{s}}+\frac{2|c| g_{m}}{R_{s}} \sqrt{\frac{\gamma \delta}{5}}\right)\left(\frac{\omega_{0}}{\omega_{T}}\right)^{2} R_{s}^{2}
\end{aligned}
$$

Substitute the operating frequencies $\omega_{01}$ and $\omega_{02}$ into (17), the corresponding noise factors can be easily got. One also can do noise optimization according to (17). After a complicated analysis and derivation process, another expressions for $F$ is obtained,

$$
F=1+a Q^{2} W^{3 / 2}+\frac{a}{4} W^{3 / 2}+b Q^{-1} W^{1 / 2}+\left(\frac{1}{d}+\frac{\gamma}{4 \alpha d}\right) Q^{-2} W^{-1 / 2}
$$

where $W$ is transistor width, $Q$ is the quality factor of the input stage network, parameters $a, b, d$ and $\alpha$ are equation coefficients, they are given by

$$
\begin{aligned}
& a=\frac{\delta \alpha\left(\frac{4}{3} \omega_{o} R_{s} C_{o x} L\right)^{2}}{R_{s} \sqrt{\frac{2 u_{e f f} C_{o x} I_{d s}}{A_{b} L}}} \\
& b=\frac{\sqrt{\frac{\gamma \delta}{4}}|c|\left(\frac{4}{3} \omega_{o} R_{s} C_{o x} L\right)}{R_{s} \sqrt{\frac{2 u_{e f f} C_{o x} I_{d s}}{A_{b} L}}} \\
& d=R_{s} \sqrt{\frac{2 u_{e f f} C_{o x} I_{d s}}{A_{b} L}} \quad \alpha=\frac{g_{m 1}}{g_{d 0}}
\end{aligned}
$$

$A_{b}$ is bulk charge factor. It is often set to one in the simplified model of the MOS transistor, but can be significantly larger. Taking derivative of (18) with

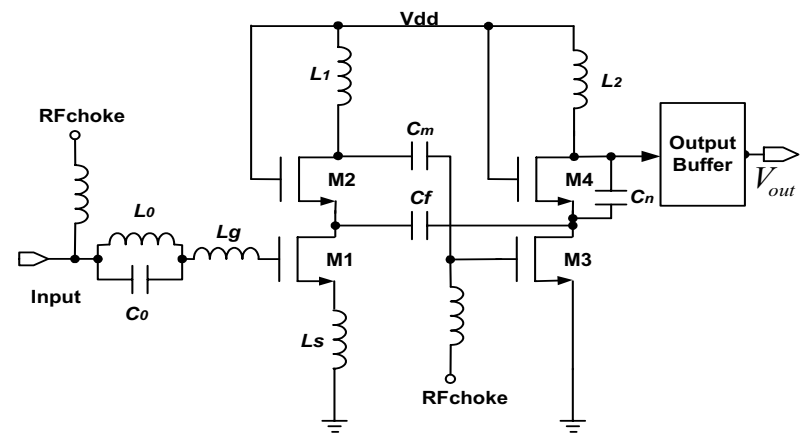

Fig. 6. Schematic diagram of the dual-band LNA respect to $W$ and equating it to zero, leads to the optimum transistor width $W_{\text {opt }}$ corresponding to the minimum $N F$,

$$
W_{o p t}=\frac{-\frac{|c|}{2} \sqrt{\frac{\gamma \delta}{4}} Q^{-1}+\frac{1}{2} Q^{-1} \sqrt{\delta\left\{\frac{\gamma|c|^{2}}{4}+3 Q^{2}(4 \alpha+\gamma)\right\}}}{4 Q^{2} \delta \alpha \omega_{o} R_{s} C_{o x} L}
$$

However, two $W_{\text {opt }}$ values will be achieved here owing to the LNA operating at two different frequencies $\omega_{01}$ and $\omega_{02}$. Thus a tradeoff between $W_{\text {opt } 1}$ and $W_{\text {opt } 2}$ has to be carried out in terms of the design requirements of different frequency bands.

\section{DUAL-BAND LNA CIRCUIT IMPLEMENTATION AND SIMULATION RESULTS}

According to the above points, a concurrent dual-band LNA is designed. The simplified schematic diagram is demonstrated in Fig. 6. The LNA is composed of an input matching stage, an intermediate stage and an output buffer stage, which is for output matching purpose. Proper tuning the passive and active devices can make the LNA work at several bands at the same time, which will be very convenient for modern multi-standard communication applications. In the circuit, cascode structure is introduced. Firstly, it is to reduce the Miller effect. Secondly, the use of a cascode improves the LNA's reverse isolation.

The designed dual-band LNA is based on IBM $0.25 \mu \mathrm{m}$ SiGe BiCMOS technology, using only CMOS devices.

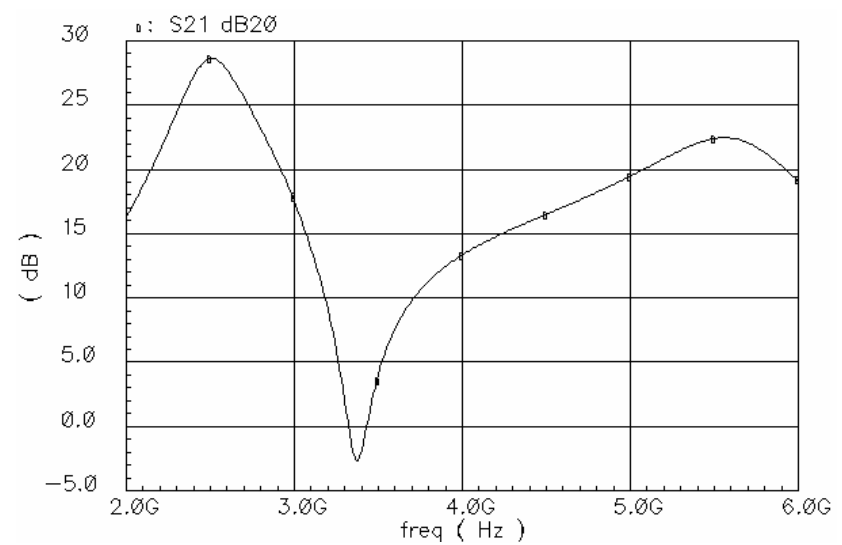

Fig. 7. Simulation result of the power gain

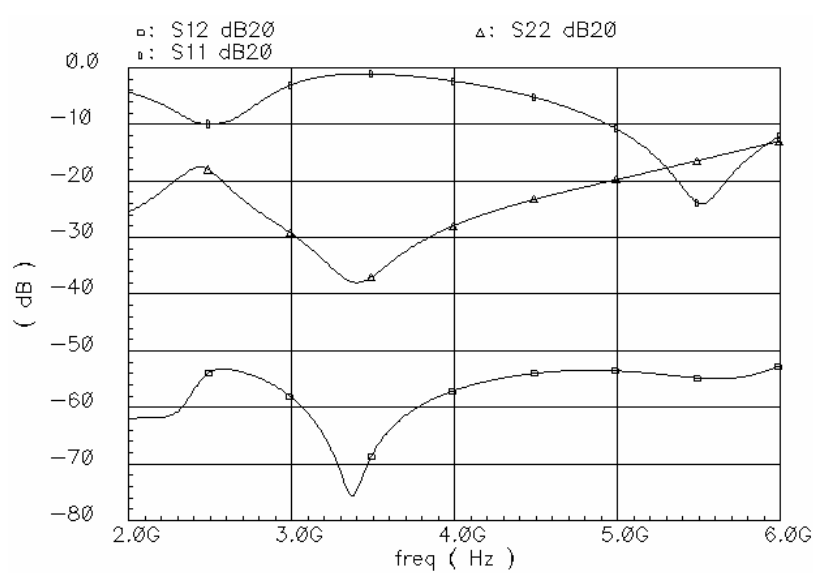

Fig. 8. Simulation results of $S_{11}, S_{12}$ and $S_{22}$ 
As known, GaAs offers the best performance. However it is costly and its back gating and side effect limit the degree of integration. Silicon, on the other hand, is

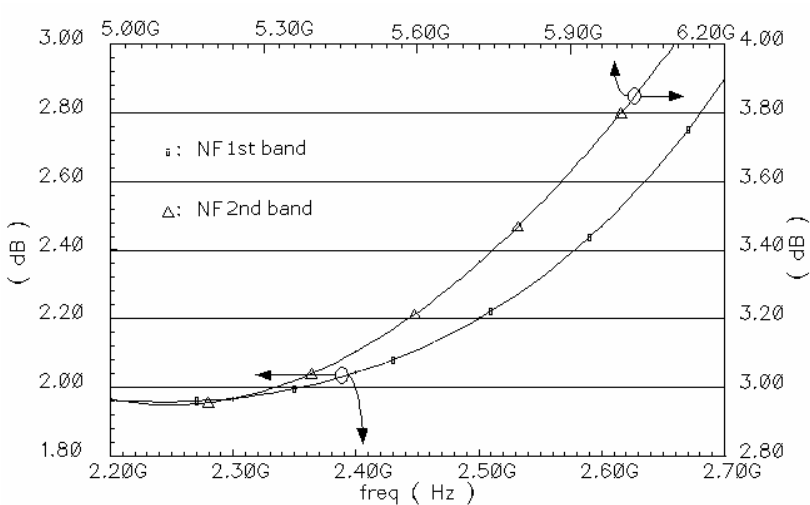

Fig. 9. Simulation result of $N F$ of the proposed dual-band LNA

abundant in nature and allows a high degree of integration. Unfortunately, silicon suffers from a lossy substrate, which restricts the quality of the on-chip passive components. While SiGe BiCMOS technology offers a comfort level (lower cost, higher integration) for silicon designers. It is especially well suited to portable wireless applications where its characteristics offer significant advantages [12].

The proposed dual-band LNA is simulated with Cadence Spectre RF. Fig. 7 - Fig. 9 illustrate the simulation results of S-parameters. The plots indicate that the LNA has a gain of about $27 \mathrm{~dB}$ at the first band and a gain about $20 \mathrm{~dB}$ at the second band. The LNA's detailed performance can be found at Table I.

TABLE I

DETAILED PERFormanCE OF THE DUAL-BAND LNA

\begin{tabular}{|c|c|c|}
\hline & $1^{\text {st }}$ band & $2^{\text {nd }}$ band \\
\hline Vdd (V) & \multicolumn{2}{|c|}{1.5} \\
\hline $\mathrm{Pd}(\mathrm{mW})$ & \multicolumn{2}{|c|}{21} \\
\hline Operating & $2.35-2.6$ & $5.0-6.0$ \\
\hline \multicolumn{3}{|l|}{ Band (GHz) } \\
\hline Standards & Bluetooth, & HiperLAN, \\
\hline Covered & IEEE $802.11 \mathrm{~b} / \mathrm{g}$ & IEEE $802.11 \mathrm{a}$ \\
\hline Gain (dB) & $26-28.6$ & $19-22$ \\
\hline $\mathrm{S}_{11}(\mathrm{~dB})$ & $-10--9$ & $-24--11$ \\
\hline $\mathrm{S}_{22}(\mathrm{~dB})$ & $-20--17.5$ & $-19.8--13$ \\
\hline $\mathrm{S}_{12}(\mathrm{~dB})$ & \multicolumn{2}{|c|}{$-60--20$} \\
\hline$N F(d B)$ & $2-2.2$ & $2.95-3.8$ \\
\hline$P_{1 \mathrm{~dB}}(\mathrm{dBm})$ & \multicolumn{2}{|c|}{ About -10} \\
\hline
\end{tabular}

\section{CONCLUSION}

A concurrent dual-band low noise amplifier for the Bluetooth, HiperLAN and Wireless LAN applications is presented in this paper. The LNA is implemented entirely on a single-chip and is possible to be integrated with other building blocks based on SiGe $0.25 \mu \mathrm{m}$ BiCMOS technology.

It has lower cost as well as better integration feasibility comparing with GaAs devices, while better performance comparing with silicon devices.

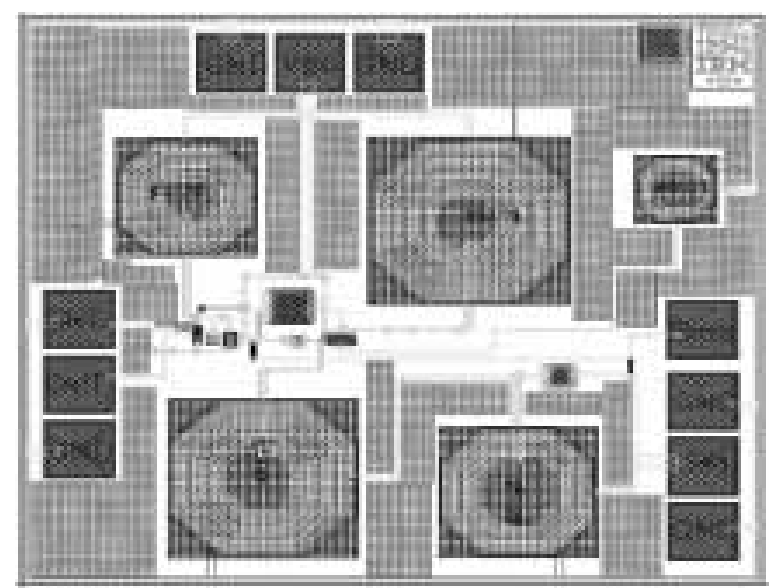

Fig. 10. Layout of the proposed dual-band LNA

\section{REFERENCES}

[1] M. J. Riezenman, "Technology 1998: analysis \& forecast communications," IEEE Spectrum, pp.29-36, January 1998

[2] Michiel S.J.Steyaert, Bram De Muer, Paul Leroux, Marc Borremans, and Koen Mertens, "Low-voltage low-power CMOS-RF transceiver design," IEEE Trans. Microwave Theory Tech., vol.50, No.1, pp.281-287, January 2002.

[3] Akira Matsuzawa, "RF-SoC-expectations and required conditions," IEEE Trans. Microwave Theory Tech., vol.50, No.1, pp.245-253, January 2002.

[4] Hassan Elwan, Hussain Alzaher and Mohammed Ismail, "A new generation of global wireless compatibility," IEEE Circuits \& Devices Magazine, vol.17, pp.7-19, January 2000.

[5] Babak Daneshrad, "Integrated circuit technologies for wireless communications," the Ninth IEEE International Symposium on Personal, Indoor and Mobile Radio Communications, pp.365-369, 1998.

[6] Jussi Ryynänen, Kalle Kivekäs, and Jarkko Jussila, “A dual-band RF front-end for WCDMA and GSM applications," IEEE J. Solid-State Circuits, vol.36, No.8, pp.1298-1204, August 2001.

[7] Stephen $\mathrm{Wu}$, and Behzad Razavi, "A 900-MHz/1.8-GHz CMOS receiver for dual-band applications," IEEE J. Solid-State Circuits, vol.33, No.12, pp.2178-2185, December 1998.

[8] Derek K. Shaeffer, "A 1.5-V, 1.5-GHz CMOS low noise amplifier," IEEE J. Solid-State Circuits, vol. 32, No.5, pp.745-759, May 1997.

[9] Peitro Andreani and Henrik Sjolland, "Noise optimization of an inductively degenerated CMOS low noise amplifier," IEEE Trans. Circuits and Systems-II: Analog and Digital Signal Processing, vol.48, No.9, pp.835-841, September 2001.

[10] Tajinder Manku, "Radio frequency CMOS", ISIC2001 tutorial material, Singapore.

[11] A. van der Ziel, Noise in Solid State Devices and Circuits. New York: Wiley, 1986.

[12] Mark Jakusoyszky and Atmel corporation, "Silicon germanium BiCMOS: who needs it?" SiGe technology, www.wirelessdesignmag.com, September 2002. 